\title{
Does cytosine-thymine-guanine (CTG) expansion size predict cardiac events and electrocardiographic progression in myotonic dystrophy?
}

\author{
N R A Clarke, A D Kelion, J Nixon, D Hilton-Jones, J C Forfar
}

\begin{abstract}
Objective-To assess whether the size of the cytosine-thymine-guanine (CTG) expansion mutation in myotonic dystrophy predicts progression of conduction system disease and cardiac events. Design-Longitudinal study involving ECG and clinical follow up over (mean (SD)) 4.8 (1.8) and 6.2 (1.9) years, respectively, of patients stratified by CTG expansion size (E0 to E4).

Patients-73 adult patients under annual review in a regional myotonic dystrophy clinic. Patients were grouped into E0/E1 $(n=25), E 2(n=34)$, and E3/E4 $(n=14)$.

Results-The proportion of patients with a QRS complex $>100 \mathrm{~ms}$ at baseline increased with the size of the CTG expansion (EO/E1, 4\%; E2, 12\%; E3/E4, 36\%; p = 0.02). This trend was more pronounced at follow up (E0/E1, 4\%; E2, 21\%; E3/E4, 57\%; p = 0.0004). The rate of widening of the QRS complex (ms/year) was similarly related to the size of the mutation (EO/E1, 0.4 (1.3); E2, 1.4 (2.5); E3/E4, $1.5(1.6) ; \mathrm{p}=0.04)$. First degree atrioventricular block was present in $23 \%$ of patients at baseline and $34 \%$ at follow up, with no significant relation to expansion size. Seven patients suffered a cardiac event during follow up (sudden death in two, permanent pacemaker insertion in three, chronic atrial arrhythmia in two), of whom six were in CTG expansion group E2 or greater. Patients who experienced a cardiac event during follow up had more rapid rates of PR interval increase (9.9 (11.1) v $1.6(2.9) \mathrm{ms} / \mathrm{year} ; \mathrm{p}=0.008)$ and a trend to greater QRS complex widening (3.6 (4.5) $v 0.9$ (1.5) ms/year; $\mathrm{p}=0.06)$ than those who did not.

Conclusions-Larger CTG expansions are associated with a higher rate of conduction disease progression and a trend to increased risk of cardiac events in myotonic dystrophy.

(Heart 2001;86:411-416)
\end{abstract}

Keywords: myotonic dystrophy; atrioventricular block; delayed intraventricular conduction; cytosine-thymine-guanine expansion

Myotonic dystrophy is a slowly progressive multisystem disorder with autosomal dominant inheritance. The disease is caused by an unstable expansion mutation of the cytosinethymine-guanine (CTG) trinucleotide repeat situated in the $3^{\prime}$ non-coding exon of a gene that encodes a serine-threonine protein kinase (DMPK). Neurological severity is positively correlated with the size of the CTG expansion mutation. ${ }^{1-4}$ The most common presentation is with neurological symptoms in the second to fourth decades of life, with more severe cases presenting neonatally (congenital myotonic dystrophy). Mild cases are often diagnosed only by kindred and molecular genetic analysis following diagnosis in another family member.

Cardiac complications including heart block and ventricular arrhythmias are well described and may occasionally be the presenting complaint. ${ }^{56}$ Sudden death is rare, and the relative importance of ventricular tachyarrhythmias and bradyarrhythmias remains unclear. ${ }^{7-11}$ Prediction of cardiac events has proved difficult, and several potential risk factors have been evaluated. There is an association between neurological severity and both electrocardiographic conduction system disease and cardiac events. ${ }^{4} 81011$ In cross sectional studies it has been found that there is a relation between the size of the CTG expansion mutation and conduction abnormalities. ${ }^{3412}$

To our knowledge, there has been no longitudinal study relating CTG expansion genotype to progression of cardiac disease. We investigated whether CTG expansion size predicts ECG progression and clinical cardiac events during long term follow up.

\section{Methods}

PATIENTS

In 1996, 90 patients with myotonic dystrophy were under annual clinic follow up in the Oxford myotonic dystrophy clinic. Seventy seven of these had serial ECG records and form the basis of our study. Four patients who had atrial arrhythmias at baseline were excluded from the analysis of ECG progression. One of these also had complete heart block at baseline. Myotonic dystrophy was diagnosed clinically by a consultant neurologist and was confirmed by an abnormal CTG expansion.

CTG TRINUCLEOTIDE EXPANSION

The CTG expansion characteristic for each patient was analysed by polymerase chain reaction (PCR) analysis for numbers of CTG repeats less than 200, and genomic analysis for larger repeats, using peripheral blood leucocytes - the accepted clinical standard for genotyping myotonic dystrophy. ${ }^{13}{ }^{14}$ The upper limit of normal for CTG expansion size is 37 repeats. CTG expansion size was classified according to the scale of Tsilfidis and colleagues: E0, 38-79; E1, 80-499; E2, 500-999; E3, $1000-1499$; E4, $\geqslant 1500 .{ }^{15}$ For the purposes of data analysis, patients in groups E0 
and $\mathrm{E} 1$ were considered together, as were those in groups E3 and E4 (one patient was classified as E0 and two as E4). The proportion of study patients in each expansion class was similar to that of the 253 patients (at April 2000) in the Oxford region molecular genetics database, drawn from a population of two million.

The size of the CTG expansion in muscle biopsy specimens is larger than in peripheral blood cells from the same patients, because of somatic heterogeneity. ${ }^{16}$

\section{NEUROLOGICAL ASSESSMENT}

The severity of skeletal muscle involvement at the baseline clinical assessment was graded on a five point scale: $1=$ no signs or only sternomastoid weakness or grip myotonia; $2=$ additional distal weakness on examination with or without symptoms; 3 = additional proximal weakness on examination with or without symptoms; $4=$ leg weakness requiring walking aids; $5=$ wheelchair bound.

ECG ANALYSIS

An ECG was recorded annually at each follow up visit. The baseline and latest follow up ECG for each patient were analysed blindly by a single experienced reporter (NC). If a cardiac arrhythmia or high degree atrioventricular (AV) block developed, the last ECG before the event was used for the assessment of conduction interval progression. The longest PR and QRS intervals from two successive sinus beats were measured to the nearest $10 \mathrm{~ms}$, and the presence or absence of bundle branch block was recorded. The following definitions were used:

- PR interval prolonged if $>200 \mathrm{~ms}$;

Table 1 Baseline clinical data

\begin{tabular}{lllllll}
\hline & & \multicolumn{3}{l}{ CTG expansion (Tsilfidis scale) } & \\
\cline { 4 - 5 } & All patients & E0/E1 & E2 & E3/E4 & p Value \\
\hline Number & 73 & 25 & 34 & 14 & \\
CTG repeats (mean (SD)) & $673(371)$ & $258(124)$ & $672(154)$ & $1249(170)$ & $<0.0001^{\star}$ \\
Age (years) (mean (SD)) & $35(14)$ & $41(13)$ & $31(13)$ & $33(15)$ & $0.03^{\star}$ \\
Male (\%) & $31(40)$ & $12(48)$ & $11(30)$ & $8(53)$ & $N^{\star}$ \\
Median neurological score (IQ range) & $2(2$ to 3) & $2(1.5$ to 3) & $2(2$ to 3$)$ & $3(2$ to 4$)$ & $0.02 \dagger$ \\
\hline
\end{tabular}

$\star$ One way analysis of variance.

†Kruskal-Wallis test.

IQ, interquartile.

Table 2 Electrocardiographic data at baseline and follow up

\begin{tabular}{|c|c|c|c|c|c|}
\hline & \multirow[b]{2}{*}{ All patients } & \multicolumn{3}{|c|}{ CTG expansion (Tsilfidis scale) } & \multirow[b]{2}{*}{$p$ Value } \\
\hline & & $E 0 / E 1$ & E2 & $E 3 / E 4$ & \\
\hline $\mathrm{n}$ & 73 & 25 & 34 & 14 & \\
\hline Follow up (years) & $4.8(1.8)$ & $5.0(1.9)$ & $4.2(1.6)$ & $5.8(1.5)$ & $0.01^{\star}$ \\
\hline PR > $200 \mathrm{~ms}$, baseline & $17(23 \%)$ & $6(24 \%)$ & $9(26 \%)$ & $2(14 \%)$ & NSt \\
\hline $\mathrm{PR}>200 \mathrm{~ms}$, follow up & $25(34 \%)$ & $7(28 \%)$ & $12(35 \%)$ & $6(43 \%)$ & $\mathrm{NS} \dagger$ \\
\hline \multicolumn{6}{|l|}{ Rate of change of PR } \\
\hline interval (ms/year) & $2.5(4.5)$ & $1.0(3.0)$ & $3.0(6.0)$ & $2.5(3.5)$ & NS $\ddagger$ \\
\hline QRS > $100 \mathrm{~ms}$, baseline & $10(14 \%)$ & $1(4 \%)$ & $4(12 \%)$ & $5(36 \%)$ & $0.02 \dagger$ \\
\hline QRS > $100 \mathrm{~ms}$, follow up & $16(22 \%)$ & $1(4 \%)$ & $7(21 \%)$ & $8(57 \%)$ & $0.0004+$ \\
\hline Rate change QRS (ms/year) & $1.1(2.1)$ & $0.4(1.3)$ & $1.4(2.5)$ & $1.5(1.6)$ & $0.04 \ddagger$ \\
\hline RBBB baseline & $2(3 \%)$ & 0 & $1(3 \%)$ & $1(7 \%)$ & NSt \\
\hline RBBB follow up & $3(4 \%)$ & 0 & $2(6 \%)$ & $1(7 \%)$ & NSt \\
\hline LBBB baseline & $1(1 \%)$ & 0 & 0 & $1(7 \%)$ & $\mathrm{NS} \dagger$ \\
\hline LBBB follow up & $3(4 \%)$ & 0 & $1(3 \%)$ & $2(14 \%)$ & NSt \\
\hline
\end{tabular}

Values are mean (SD) or $\mathrm{n}(\%)$.

${ }^{\star}$ One way analysis of variance.

$\dagger \chi^{2}$ test.

$\neq$ Kruskal-Wallis test.

LBBB, left bundle branch block; RBBB, right bundle branch block.
- complete right bundle branch block if typical QRS morphology of at least $120 \mathrm{~ms}$;

- complete left bundle branch block if typical QRS morphology of at least $120 \mathrm{~ms}$;

- partial left or right bundle branch block if typical QRS morphology of between 100$120 \mathrm{~ms}$;

- non-specific QRS prolongation if QRS duration greater than 100 ms but not meeting any of the above criteria.

Twenty four hour Holter monitoring was performed only in patients with symptoms suggestive of a tachyarrhythmia or bradyarrhythmia.

CARDIAC EVENTS

The following cardiac events were documented during follow up: tachyarrhythmias (chronic or paroxysmal atrial arrhythmias, ventricular tachycardia); pacemaker implantation; sudden cardiac death. Echocardiography was not routinely performed except in patients with atrial or ventricular arrhythmias and those with cardiac symptoms.

\section{STATISTICAL ANALYSIS}

Data are expressed mean (SD) unless otherwise stated. Continuous variables were compared between the three CTG expansion groups using one way analysis of variance (ANOVA), or non-parametric ANOVA with the Kruskal-Wallis test where there was a significant deviation from the normal distribution. The rate of change of PR and QRS intervals was compared between patients with and without a cardiac event using a Mann-Whitney $\mathrm{U}$ test. Categorical variables were compared using the $\chi^{2}$ test. A probability value of $\mathrm{p}<0.05$ was assumed to be significant.

\section{Results}

BASELINE CLINICAL CHARACTERISTICS

Table 1 shows the baseline clinical characteristics of the 73 patients, stratified by CTG expansion group. Thirty three patients were from 13 kindreds, and 40 were unrelated. Those with larger expansions tended to present at an earlier age and have more severe neurological disease.

ELECTROCARDIOGRAPHIC ANALYSIS

These results are shown in table 2 . The mean duration of ECG follow up was 4.8 (1.8) years for all patients. Those in expansion group $\mathrm{E} 3 / \mathrm{E} 4$ tended to be followed for longer than those in group E2.

Atrioventricular conduction

At baseline, 17 of the 73 patients (23\%) had first degree AV block, and this increased to 25 $(34 \%)$ at follow up. The mean rate of increase in PR interval for all patients was 2.5 (4.5) $\mathrm{ms} /$ year, with no significant differences between CTG groups.

\section{Intraventricular conduction}

At baseline, 10 patients (14\%) had a QRS duration greater than $100 \mathrm{~ms}$, and the number increased to $16(22 \%)$ at follow up. Patients with larger CTG expansions were more likely 


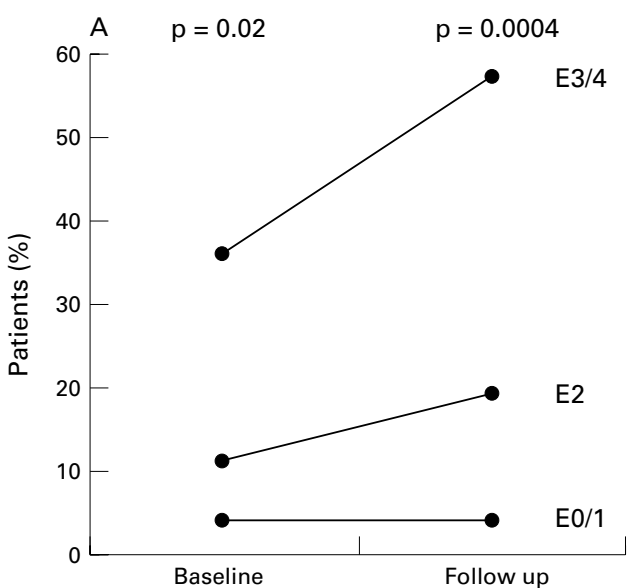

Figure 1 Effect of CTG expansion group on $(A)$ prevalence of intraventricular conduction delay $(\mathrm{ORS}>$ $100 \mathrm{~ms}$ ) at baseline and follow up, and (B) mean (SEM) rate of progression of intraventricular conduction delay. Comparison in (A) using $\chi^{2}$ test, in (B) using Kruskal-Wallis test.

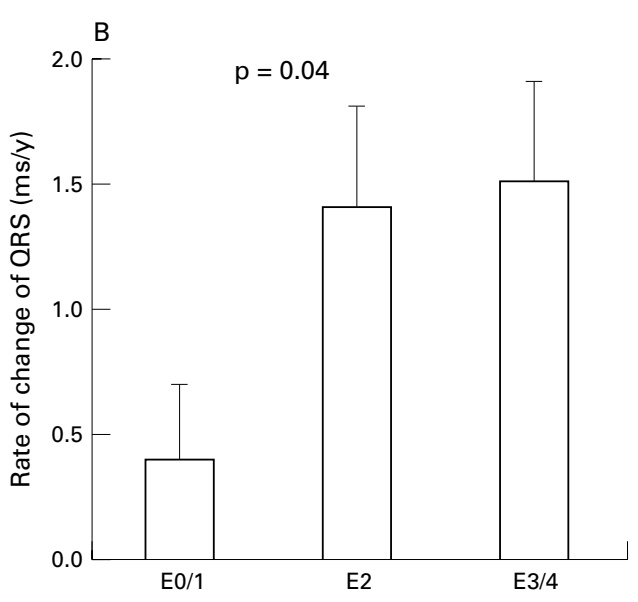

Table 3 Cardiac events occuring in 11 of 77 patients

\begin{tabular}{llllll}
\hline Patient & Sex & Age at first event (years) & CTG code & Time to event (years) & Event \\
\hline 1 & M & 62 & E1 & 6.0 & CAA \\
2 & F & 41 & E2 & Baseline & CAA \\
3 & M & 18 & E2 & Baseline & CAA \\
4 & M & 25 & E2 & Baseline & CAA \\
5 & F & 58 & E2 & 1.3 & PPM \\
6 & M & 67 & E2 & 3.1 & CAA \\
7 & F & 54 & E2 & 4.6 & PPM \\
8 & F & 36 & E2 & 4.0 & CAA \\
& & & & 5.4 & PPM \\
9 & M & 44 & E3 & Baseline & CAA, PPM \\
10 & F & 34 & E3 & Baseline & PAF \\
& & & & 4.1 & VT \\
11 & M & 28 & E3 & 5.4 & SCD \\
& & & & 9.3 & PPM \\
& & & & 9.3 & SCD
\end{tabular}

CAA, chronic atrial arrhythmia; PPM, permanent pacemaker; SCD, sudden or arrhythmic cardiac death; VT, ventricular tachycardia. Those with atrial arrhythmias at baseline (patients $2,3,4$, and 9) were excluded from the analysis of conduction disease progression.

Table 4 Cardiac events stratified by CTG expansion group

\begin{tabular}{|c|c|c|c|c|c|}
\hline & \multirow[b]{2}{*}{ Total } & \multicolumn{3}{|c|}{ CTG expansion (Tsilfidis scale) } & \multirow[b]{2}{*}{$p$ Value } \\
\hline & & $E 0 / E 1$ & E2 & $E 3 / E 4$ & \\
\hline $\mathrm{n}$ & 77 & 25 & 37 & 15 & \\
\hline $\begin{array}{l}\text { Follow up (years) } \\
\text { (mean (SD)) }\end{array}$ & $6.2(1.9)$ & $6.2(2.4)$ & $5.9(1.6)$ & $7.1(1.3)$ & 0.01 \\
\hline CAA & 5 & 1 & 4 & 0 & \\
\hline PPM & 4 & 0 & 3 & 1 & \\
\hline VT & 0 & 0 & 0 & 0 & \\
\hline SCD & 2 & 0 & 0 & 2 & \\
\hline Total (\%) & $11(14)$ & $1(4)$ & $7(19)$ & $3(20)$ & $\mathrm{NS}^{\star}$ \\
\hline
\end{tabular}

Where patients had more than one event, only the one placed highest in the following hierarchy was included: SCD (sudden or arrhythmic death) > VT (ventricular tachycardia) > PPM (permanent pacemaker) $>$ CAA (chronic atrial arrhythmia).

${ }^{\star} \chi^{2}$ test. to show an intraventricular conduction delay at baseline, and this trend was more pronounced at follow up (table 2 and fig 1 ). The mean rate of increase of the QRS interval was 1.1 (2.1) $\mathrm{ms} /$ year for all patients. Patients in CTG group E2 or larger showed a greater progression than those in group E0/E1 (table 2 and fig 1). The majority of patients with QRS prolongation had a non-specific intraventricular conduction delay. Only three had bundle branch block at baseline, and a further three developed it during follow up (table 2).

CARDIAC EVENTS

Cardiac events are shown in tables 3 and 4 . During follow up there were nine deaths. Seven were from non-cardiac causes (pneumonia and respiratory failure in four, and one case each of abdominal sepsis, postoperative pulmonary embolism, and suicide). Two patients in CTG class E3 suffered a sudden cardiac death.

The mean duration of clinical follow up was 6.2 (1.9) years for all patients. Those in expansion group E3/E4 underwent longer follow up than those in group E2. Eleven patients experienced 16 cardiac events. Five patients had cardiac events at baseline, and this was the presenting complaint in two (patients 3 and 9). Seven patients developed 11 cardiac events during follow up. For patients who suffered more than one cardiac event, the most significant was recorded using the following hierarchy: sudden or arrhythmic death (SCD) $>$ ventricular tachycardia $(V T)>$ permanent pacemaker $(\mathrm{PPM})>$ chronic atrial arrhythmia (CAA). The majority of events occurred in group E2 or above, and there was a nonsignificant trend towards more serious events with larger CTG expansions.

RELATION BETWEEN RATE OF ECG PROGRESSION AND CARDIAC EVENTS

Seven of the 24 patients with first degree AV block (PR interval $>200 \mathrm{~ms}$ ) or delayed intraventricular conduction (QRS > $100 \mathrm{~ms}$ ) experienced a cardiac event during follow up, compared with none of the 49 with normal conduction intervals $(p<0.0001)$. The mean follow up period for the two groups was not significantly different, at 6.8 (2.0) and 5.9 (1.8) years, respectively. Patients who experienced a cardiac event during follow up had more rapid rates of PR increase (9.9 (11.1) v $1.6(2.9) \mathrm{ms} /$ year; $p=0.008)$ and a trend to more rapid rates of QRS increase (3.6 (4.5) v 0.9 (1.5) ms/year; $\mathrm{p}=0.06)$ than those who did not.

\section{ATRIAL ARRHYTHMIAS}

Of the eight patients with clinically significant atrial arrhythmias, five had persistent atrial flutter, one persistent atrial fibrillation, one paroxysmal atrial fibrillation, and one persistent atrial tachycardia. Three of these patients who had a fast ventricular rate were symptomatic; of these, two in group E2 underwent successful radiofrequency ablation for type 1 atrial flutter and one had prolonged episodes of paroxysmal atrial fibrillation. 
PERMANENT PACEMAKER IMPLANTATION

Five patients underwent permanent pacemaker implantation, one performed at baseline. The indication was recurrent syncope in four, in whom two had complete AV block (one at baseline, one during follow up), one had intermittent Mobitz 2 AV block, and one had widespread conduction system disease. Prophylactic pacemaker implantation was performed in the patient with widespread progressive conduction system disease (PR interval 230$250 \mathrm{~ms}$ and QRS 100-160 ms over a five year follow up).

VENTRICULAR ARRHYTHMIAS AND SUDDEN DEATH Symptomatic sustained ventricular tachycardia occurred in two siblings in CTG expansion group E3. The first (patient 11 in table 3) presented as an emergency with ventricular tachycardia and hypotension unresponsive to amiodarone and cardioversion. He died following degeneration to ventricular fibrillation. His sister (patient 10 in table 3) experienced recurrent symptomatic ventricular tachycardia and paroxysmal atrial fibrillation. She died suddenly at home despite treatment with amiodarone. Another brother had first degree AV block and a prolonged QRS interval. Their mother, who was not in the study, required a permanent pacemaker for recurrent syncope with documented first degree heart block, left bundle branch block, and sinus pauses. All three siblings and their mother had a PR interval greater than $210 \mathrm{~ms}$ and a QRS interval greater than $110 \mathrm{~ms}$.

\section{ECHOCARDIOGRAPHY}

Echocardiography was performed in five of eight patients with atrial arrhythmias. The study was entirely normal in four and all had a left atrial size of $\leqslant 3.8 \mathrm{~cm}$. The fifth patientwho also had episodes of sustained ventricular tachycardia and suffered sudden cardiac death-had mild anterior mitral valve leaflet prolapse, mild concentric left ventricular hypertrophy, and normal coronary arteries on angiography. The other patient with ventricular tachycardia and sudden cardiac death presented as an emergency and died before echocardiography could be performed.

\section{Discussion}

To our knowledge this is the first longitudinal study showing that ECG progression is related to the size of the CTG expansion in myotonic dystrophy. Patients with larger expansions were at increased risk of intraventricular conduction disease at baseline, and showed more rapid progression during follow up. The frequency of cardiac events increased with larger expansion size although significance was not reached owing to the small numbers involved.

\section{STUDY POPULATION}

The 253 patients with myotonic dystrophy currently on the Oxford region molecular genetics database yield a population prevalence of 1 in 8000, similar to previous estimates. ${ }^{17}$ Our study population was relatively unselected as the decision to undertake follow up was determined geographically and was independent of clinical severity. The distribution of CTG expansion sizes was comparable with that of the Oxford region molecular genetics database as a whole. In agreement with several other studies we found a significant relation between neurological severity, age at presentation, and CTG expansion size..$^{1-315} 18$

\section{ECG ABNORMALITIES}

Although first degree AV block was common, the rate of increase of PR interval was small in this and previous studies. ${ }^{8919}$ The mean value for our patients was 2.5 (4.5) $\mathrm{ms} /$ year, or $1.2 \%$ per year, similar to that of $1.5 \%$ found by Hawley and colleagues. ${ }^{8}$

Patients with larger CTG expansions were more likely to have prolonged QRS complexes at baseline, and had higher rates of increase during follow up. A previous report found no correlation between conduction disease and mutation size. ${ }^{18}$ However, this was a selected population, as $87 \%$ of the patients had at least first degree AV block or symptoms suggestive of bradycardia. In the majority of patients in our study, QRS prolongation was caused by non-specific intraventricular conduction delay, with complete bundle branch block in only $8 \%$ of all patients at follow up. Melacini and colleagues reported a $75 \%$ prevalence of bundle branch block in the E3/E4 group ${ }^{3}$ compared with the $21 \%$ in our study. Some of this discrepancy can be explained by differences in study populations, as $80 \%$ of Melacini's E3/E4 group had severe neurological disease whereas $29 \%$ of patients in E3/E4 in our study had a neurological score of 4 or 5 at baseline. Differences in the ECG criteria used may also have contributed as those investigators did not comment on non-specific intraventricular conduction delay. Others have reported complete bundle branch block in $18-26 \%$ of patients in both cross sectional and follow up studies. $^{3} 41019$

\section{CARDIAC EVENTS}

Both the baseline ECG and the mutation expansion size predicted future cardiac events. When the incidence of cardiac events was assessed between the predetermined expansion groups there was no significant difference, although 10 of the 11 patients who suffered a cardiac event had an $\mathrm{E}$ code of 2 or above. Those with higher $\mathrm{E}$ codes were younger at presentation and had more severe neurological involvement, consistent with the previous reports noted above. It is likely that larger CTG expansions have a greater pathological effect on the heart earlier in life and cause a greater rate of deterioration, in a similar way to the effect on skeletal muscle. Cardiac events during follow up only occurred in patients with abnormal conduction at baseline. Those experiencing cardiac events also had a significantly higher rate of $P R$ interval progression, although the higher rate of QRS progression just failed to reach significance. In a previous longitudinal study $85 \%$ of all events occurred in those with a baseline PR interval of $240 \mathrm{~ms}$ or greater. ${ }^{10}$

Prognosis in myotonic dystrophy is mainly determined by the severity of the neurological 
disease which is associated with death from respiratory failure and pneumonia. ${ }^{20}$ In the series of Colleran and colleagues, ${ }^{10}$ six of the seven deaths were respiratory, compared with four of nine in our study. Sudden death is well recognised, but is rare and difficult to predict, with most studies reporting only one case..$^{7-10} 19$ The two sudden deaths in our study equate to a risk of $0.4 \%$ per patient-year, similar to the $0.2 \%$ per patient-year in another cohort of 367 patients followed for 10 years. ${ }^{20}$ The prolongation of total QRS duration on signal averaged electrocardiography (SAECG) in myotonic dystrophy is primarily related to delayed activation of the His and Purkinje tissue and not to the development of true late potentials. Therefore SAECG is not useful for identifying patients at increased risk of ventricular arrhythmias. ${ }^{21}$ Conduction system disease is common in myotonic dystrophy, but the significance of $\mathrm{AV}$ block as a mechanism of sudden death remains unclear. Conduction system disease is most prominent distally, and AV block is usually infrahisian. ${ }^{18}{ }^{22}$

Prolongation of the bundle of His to ventricle (HV) interval can be inferred from SAECG, or may be measured directly at electrophysiological study, ${ }^{12}{ }^{18}$ but is a poor prognostic marker because of its high prevalence and the low rate of cardiac events. Lazarus and colleagues found a high incidence (39\% over a two year follow up period) of asymptomatic paroxysmal complete AV block in patients with a prolonged $\mathrm{HV}$ interval implanted with a diagnostic DDDR permanent pacemaker, but pacemaker implantation for persistent or symptomatic complete AV block is relatively infrequent. ${ }^{23}$ Three $(4.0 \%)$ of the patients in our study received a permanent pacemaker during follow up for recurrent syncope and either high degree AV block or widespread conduction disease. In previous prospective studies with a mean follow up of 4-6 years cumulative pacing frequency varied between $2.7-16 \%$, but overall only $3 \%$ of the patients had documented symptomatic bradycardia or high degree AV block, comparable to the value in our study. ${ }^{8-10} 19$ In a recent large prospective study, 13 of 367 patients $(3.5 \%)$ required a pacemaker for complete heart block in a 10 year follow up period. ${ }^{20}$ It is likely that the threshold for pacemaker insertion varies between centres because of the poorly understood mechanism of sudden death.

Although conduction system disease is common, its main significance may be as a marker for ventricular arrhythmias. There are occasional reports of sudden cardiac death in the presence of a normally functioning pacemaker. ${ }^{519}$ All of the paced patients in our study presented either with a stable ventricular escape rhythm or had slowly progressive conduction disease before they developed symptoms. In contrast, ventricular arrhythmias may cause greater haemodynamic instability and be more unpredictable and difficult to treat. Possible mechanisms of ventricular tachycardia in myotonic dystrophy include ventricular re-entry secondary to myocardial fibrosis, torsade de pointes in association with high degree AV block, and bundle branch re-entry. ${ }^{24-27}$ In our study both the cardiac deaths were unequivocally caused by ventricular tachyarrhythmias. Several family members had widespread conduction disease. Others have reported clustering of cardiac events in families, some of which show a particular risk of sudden death. ${ }^{28} 29$ There is a suggestion that the more serious events occurred in the E3/E4 group, although the number of events is too small to allow significance, and both patients with ventricular tachycardia came from one family.

Atrial arrhythmias occurred in eight of 11 patients who had cardiac events, with atrial flutter being the most common as has been reported previously. ${ }^{30}$ The majority of these patients were asymptomatic owing to a combination of exercise limitation secondary to neurological disease and a relatively well controlled ventricular rate in the absence of AV node blocking drugs.

Echocardiography was not routinely performed in our study, except in patients with atrial and ventricular arrhythmias. Previous studies have shown either no impairment of left ventricular function or mild impairment of systolic or diastolic function, clinical heart failure being very rare. ${ }^{341731}$

\section{CONCLUSIONS}

We have shown that larger CTG expansions predict a higher rate of QRS broadening and a trend towards a higher incidence of cardiac events. Those experiencing cardiac events during follow up had a significantly higher rate of PR interval progression.

A normal ECG predicts a low risk of cardiac events in the medium term. Sudden death is uncommon, but individuals with a family history of sudden cardiac death may be at particularly high risk. In rare instances, electrophysiological evaluation of electrical stability and even cardioverter-defibrillator implantation may be justified. An annual ECG combined with an evaluation of cardiac symptoms is adequate in the majority of patients in whom the progression of cardiac disease is slow.

We thank Dr Gail Norbury, molecular geneticist at Oxford Medical Genetics Laboratories, for gene typing and access to all genetic analysis results in the Oxforshire region; and Dr Susan Huson, consultant clinical geneticist, who-in conjunction with Anne Roberts, clinical nurse specialist in genetics-followed up and assessed patients with myotonic dystrophy acquired through referral and kindred analysis.

1 Harley HG, Rundle SA, MacMillan JC, et al. Size of the unstable CTG repeat sequence in relation to phenotype and parental transmission in myotonic dystrophy. $A m$ f Hum Genet 1993;52:1164-74.

2 Ashizawa T, Dubel JR, Dunne PW, et al. Anticipation in myotonic dystrophy. II. Complex relationships between clinical findings and the structure of the CTG repeat. Neurology 1992;42:1877-83.

3 Melacini P, Villanova C, Menegazzo E, et al. Correlation between cardiac involvement and CTG trinucleotide repeat length in myotonic dystrophy. $\mathcal{F} \mathrm{Am}$ Coll Cardiol 1995;25:239-45.

4 Tokgozoglu LS, Ashizawa T, Pacifico A, et al. Cardiac involvement in a large kindred with myotonic dystrophy. Quantitative assessment and relation to size of CTG expansion. FAMA 1995;274:813-19.

5 Grigg LE, Chan W, Mond HG, et al. Ventricular tachycardia and sudden death in myotonic dystrophy: clinical, electrophysiologic and pathologic features. $\mathcal{f} \mathrm{Am}$ Coll Cardiol physiologic and

6 Bache RJ, Sarosi GA. Myotonia atrophica: diagnosis in patient with complete heart block and Stokes-Adams syncope. Arch Intern Med 1968;121:369-72. 
7 Moorman JR, Coleman RE, Packer DL, et al. Cardiac involvement in myotonic muscular dystrophy. Medicine involvement in myotonic m.
(Baltimore) 1985;64:371-87.

8 Hawley RJ, Milner MR, Gottdiener JS, et al. Myotonic heart disease. A clinical follow-up. Neurology 1991;41:259-62.

9 Fragola PV, Luzi M, Calo L, et al. Cardiac involvement in myotonic dystrophy. Am F Cardiol 1994;74:1070-2.

10 Colleran JA, Hawley RJ, Pinnow EE, et al. Value of the electrocardiogram in determining cardiac events and mortality in myotonic dystrophy. Am f Cardiol 1997;80:1494-7.

11 Olofsson B-O, Forsberg H, Andersson S, et al. Electrocardiographic findings in myotonic dystrophy. $\mathrm{Br}$ Heart $\mathcal{f}$ 1988;59:47-52.

12 Babuty D, Fauchier L, Tena-Carbi D, et al. Is it possible to identify infrahisian cardiac conduction abnormalities in myotonic dystrophy by non-invasive methods? Heart 1999; 82:634-7.

13 Mahadevan M, Tsilfidis C, Sabourin L, et al. Myotonic dystrophy mutation: an unstable CTG repeat in the 3 trophy mutation: an unstable CTG repeat in the

14 Aslanidis C, Jansen G, Amemiya C, et al. Cloning of the essential myotonic dystrophy region and mapping of the putative defect. Nature 1992;355:548-51.

15 Tsilfidis C, Mackenzie AE, Mettler G, et al. Correlation between CTG trinucleotide repeat length and frequency of severe congenital myotonic dystrophy. Nat Genet 1992;1 $192-5$

16 Anvret M, Ahlberg G, Grandell U, et al. Larger expansions of the CTG repeat in muscle compared to lymphocytes from patients with myotonic dystrophy. Hum Mol Genet 1993;2:1397-400.

17 Phillips MF, Harper PS. Cardiac disease in myotonic dystrophy. Cardiovasc Res 1997;33:13-22.

18 Lazarus A, Varin J, Ounnoughene Z, et al. Relationships among electrophysiological findings and clinical status, heart function, and extent of DNA mutation in myotonic dystrophy. Circulation 1999;99:1041-6.
19 Florek RC, Triffon DW, Mann DE, et al. Electrocardiographic abnormalities in patients with myotonic dystrophy. West $\mathcal{F}$ Med 1990;153:24-7.

20 Mathieu J, Allard P, Potvin L, et al. A 10-year study of mortality in a cohort of patients with myotonic dystrophy. Neurology 1999;52:1658-62.

21 Babuty D, Fauchier L, Tena-Carbi D, et al. Significance of late potentials in myotonic dystrophy. $\mathrm{Am} \mathcal{F}$ Cardiol 1999;84:1099-101

22 Nguyen HH, Wolfe JT, Holmes DR, et al. Pathology of the cardiac conduction system in myotonic dystrophy: a study of 12 cases. 7 Am Coll Cardiol 1988;11:662-71.

23 Lazarus A, Babuty D, Victor J, et al. Multicentre study about sudden death in myotonic dystrophy: results at two years. PACE 1998;21:981.

24 Litchfield JA. A-V dissociation in dystrophia myotonica. $\mathrm{Br}$ Heart F 1953;15:357-9.

25 Strasberg B, Kanakis C, Dhingra RC, et al. Myotonia dystrophia and mitral valve prolapse. Chest $1980 ; 78: 845-8$.

26 Umeda Y, Ikeda U, Yamamoto J, et al. Myotonic dystrophy associated with QT prolongation and torsades de pointes. Clin Cardiol 1999;22:136-8.

27 Merino JL, Carmona JR, Fernandez-Lozano I, et al. Mechanisms of sustained ventricular tachycardia in myotonic dystrophy: implications for catheter ablation. Circulation 1998;98:541-6.

28 Hawley RJ, Gottdiener JS, Gay JA, et al. Families with myotonic dystrophy with and without cardiac involvement. Arch Intern Med 1983;143:2134-6.

29 Melillo G, Ruggieri MP, Magni G, et al. Malignant cardiac involvement in a family with myotonic dystrophy. G Ital Cardiol 1996;26:853-61.

30 Church SC. The heart in myotonia atrophica. Arch Intern Med 1967;119:176-81.

31 Hayashi Y, Ikeda U, Kojo T, et al. Cardiac abnormalities and cytosine-thymine-guanine trinucleotide repeats in myotonic dystrophy. Am Heart f 1997;134:292-7.

\section{IMAGES IN CARDIOLOGY}

\section{Endomysium in left ventricle}

This image shows the three dimensional arrangement of endomysial collagen fibres in the left ventricle free wall. The endomysium was studied by means of scanning electron microscopy after sodium hydroxide maceration in rabbit non-contracted heart. This method of maceration allowed the isolation of collagen fibres, the elimination of all other tissue elements, and the preservation of endomysial structure and position. The technique allowed the visualisation of a continuous three dimensional network of collagen fibres that delimited wide and empty lacunae. The morphological evaluation showed that this network consisted of endomysial fibres that enwrapped the contiguous spaces devoid of myocytes (muscular lacunae $(\mathrm{ml})$ ) and capillaries (capillary lacunae (cl)) that were eliminated by the maceration $(\times 2000$, bar $=10 \mu \mathrm{m})$. This thin web appeared as a continuous structure that completely separated one myocyte from another and enveloped both myocytes and capillaries. This image also showed that the collagen connections distributed among heart capillaries and myocytes were not only represented by isolated twisted struts, as usually described, but were actually formed by laminae that extended over the entire cell surface. This study also allowed the observation of the struts that were often reported in previous studies performed using other microscopic techniques.

The peculiar three dimensional architecture shown in this image ensures protection against

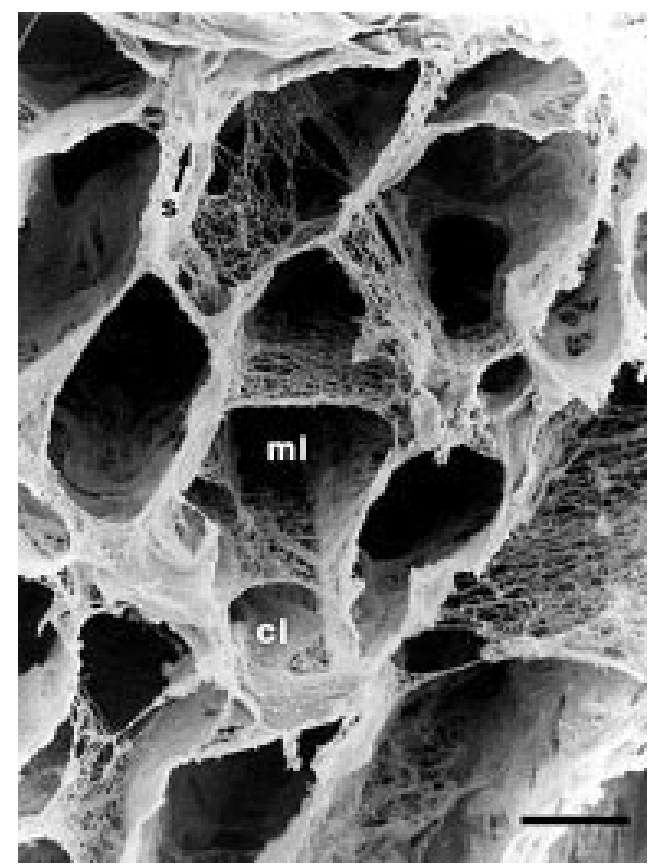

myocyte overstretch, and may provide one of the morphological bases of cardiac muscular compliance.

GUIDO MACCHIARELLI OSAMU OHTANI guido.macchiarelli@uniroma1.it 